\title{
Incidence du Yam mosaic virus (YMV) et du Cucumber mosaic virus (CMV) sur des variétés de Dioscorea spp. cultivées dans les régions de Bouaké et de Toumodi en Côte d'Ivoire
}

\author{
K. SEKA ${ }^{1 *}$, A. H. DIALLO ${ }^{1}$, N. K. KOUASSI ${ }^{2}$ et S. AKE ${ }^{3}$ \\ ${ }^{1}$ Université d'Abobo-Adjamé. UFR des Sciences de la Nature. Laboratoire de Phytopathologie. \\ 02 BP 801 Abidjan 02, Côte d'Ivoire. \\ ${ }^{1}$ Université d'Abobo-Adjamé. UFR des Sciences de la Nature. Laboratoire de Phytopathologie. \\ 02 BP 801 Abidjan 02, Côte d'Ivoire. \\ ${ }^{2}$ CNRA, Laboratoire Central de Biotechnologies, KM 17 route de Dabou \\ 01 BP 1740 Abidjan 01, Côte d'Ivoire. \\ ${ }^{3}$ Université de Cocody. UFR de Biosciences, 22 BP 582 Abidjan 22, Côte d'Ivoire. \\ *Auteur correspondant, E-mail: sekoutoua@yahoo.fr, Tél.: (225) 05636172.
}

\section{RESUME}

L'évolution de la distribution et de l'incidence du YMV et du CMV infectant les ignames en Côte d'Ivoire et les pertes de rendement qu'ils occasionnent nécessitent un état des lieux de leur présence. Cette étude a pour objectif de déterminer la distribution et l'incidence du YMV et du CMV infectant cette plante à tubercule dans les deux (2) zones majeures de production (Toumodi et Bouaké) en Côte d'Ivoire. A cet effet, une identification et une caractérisation de ces deux (2) virus ont été réalisés sur 18 parcelles paysannes reparties dans trois (3) localités de chacune des zones. Les tests de détection (TAS-ELISA pour le YMV; TASELISA et DAS-ELISA pour le CMV) réalisés sur 1152 échantillons dont 576 feuilles, 288 tiges et 288 tubercules ont confirmé la présence de ces virus. Ainsi, la mosaïque, le shoestring, et le gaufrage des feuilles sont causés par le YMV tandis que les chloroses sont provoquées uniquement par le CMV. Ces virus sont présents dans la zone de Bouaké et de Toumodi avec respectivement des incidences moyennes de $88 \%$ et $95 \%$ pour le YMV et de 10,85\% et 16,28\% pour le CMV. Des infections mixtes $(4,10 \%)$ de YMV et CMV ont été observées sur Bètè-bètè, Florido et Lokpa.

(C) 2009 International Formulae Group. All rights reserved.

Mots clés: YMV; CMV; Distribution, Incidence; tests ELISA; Infection mixte.

\section{INTRODUCTION}

Les ignames (Dioscorea spp.) jouent un rôle important dans l'alimentation et les activités socioculturelles en Côte d'Ivoire (FAO, 2007). Elles constituent également une source de revenus non négligeable pour des milliers de personnes vivant dans le monde. Les pays de l'Afrique de l'Ouest et du Centre contribuent pour plus de $90 \%$ à la production annuelle mondiale évaluée à 38 millions de tonnes d'ignames (FAO, 2007). Malheureusement la grande sensibilité des espèces de Dioscorea aux virus suivants, Yam Mosaic Virus (YMV) et Cucumber Mosaic Virus (CMV), constitue l'une des préoccupations majeures des producteurs d'ignames en Côte d'Ivoire et ailleurs en Afrique (Odu et al., 2004). En effet, ces deux (2) virus affectent sérieusement les rendements de cette culture (Thouvenel et Dumont, 1990; Asiedu et al., 1998 ; Eni et al., 2008a; Jones et al., 2008 ; Séka et al., 2009). Thouvenel et al. (1989) et Kouamé et al. (2003) ont montré que dans la zone savanicole 
de la Côte d'Ivoire, l'incidence du YMV et du CMV diffère de celle observée en zone forestière. La caractérisation moléculaire du YMV et CMV a été déjà réalisée dans d'autres pays (Bousalem et al., 2000). L'incidence du YMV et du CMV pourrait varier en fonction des différentes variétés de Dioscorea spp. et des deux grandes zones de production d'ignames (Gumedzoe et al., 2003) que sont Bouaké (zone de savane arborée) et Toumodi (zone de transition forêt/savane arborée) (Séka et al., 2009). Compte tenu de l'accroissement des symptômes de viroses dans les champs d'ignames (Odu et al., 2004 ; Eni et al., 2008a ; Eni et al., 2008b) et des pertes de rendement qu'occasionnent ces virus (Lebas, 2002; Séka et al., 2009), il est donc nécessaire d'inventorier et de caractériser les agents responsables de ces viroses dans les cultures d'ignames des deux principales zones de production d'ignames en Côte d'Ivoire. Cette étude porte sur la distribution et l'incidence du YMV et du CMV en fonction des espèces et/ou variétés; du stade phénologique de la plante ainsi que des localités et zones d'étude.

\section{MATERIEL ET METHODES \\ Sites d'étude}

Cette étude de distribution et d'incidence du YMV et du CMV de l'igname en Côte d'Ivoire a été menée dans 3 localités de chacune des zones de production que sont Bouaké et Toumodi.

\section{Zone de Bouaké}

La zone de Bouaké est située sur la latitude $7^{\circ} 69^{\prime} \mathrm{N}$ et la longitude $5^{\circ} 03^{\prime} \mathrm{O}$ (Figure 1). La végétation est constituée de savane arborée avec plusieurs espèces de poacées. Les sols présentent une texture sablo argileuse. Le climat est de type tropical humide avec deux saisons de pluie de mai à novembre et deux saisons sèches de novembre à mai. Les précipitations moyennes annuelles sont de $1200 \mathrm{~mm}$ de pluie.

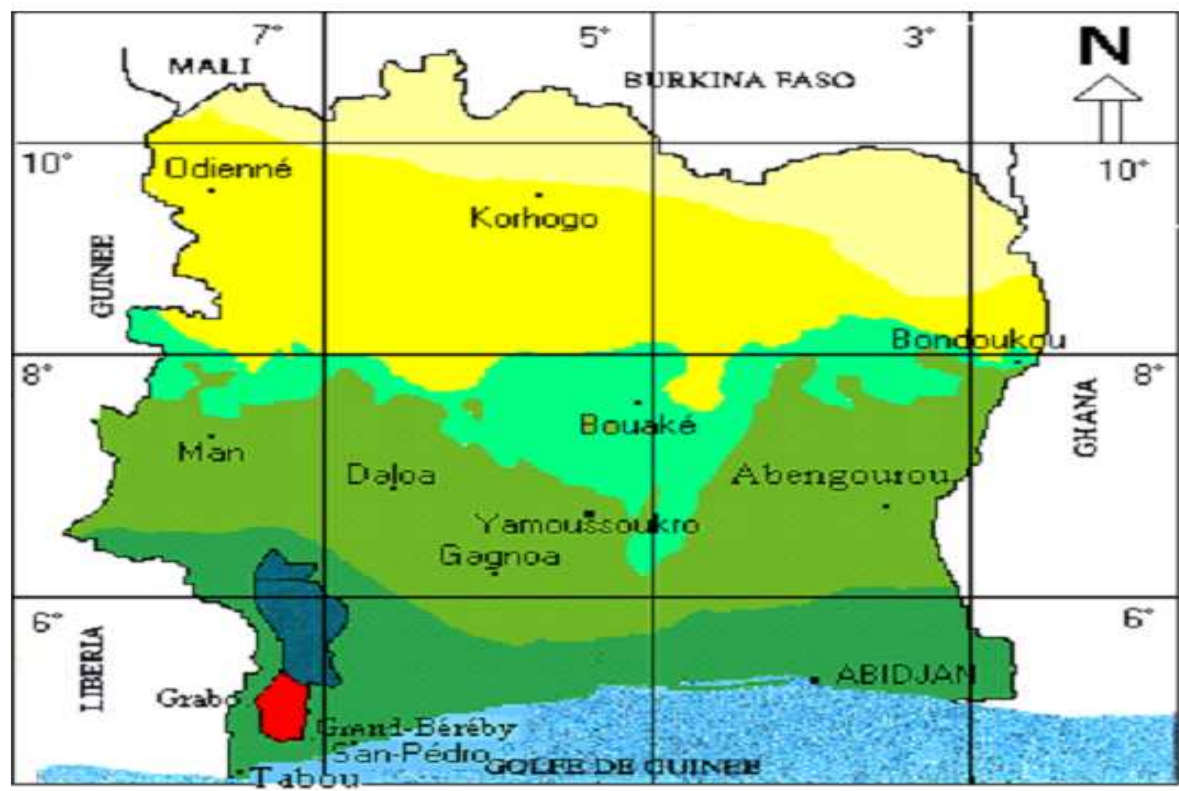

Echelle 1/4000000

Zones d'étude Savane herbeuse
Forêt dense Savane soudanienne
Forêt claire Forêt classée
Savane arborée

Forêt classée

Figure 1: Répartition de la végétation en Côte d'Ivoire. 


\section{Zone de Toumodi}

La zone de Toumodi est située sur la latitude $6^{\circ} 55^{\prime} \mathrm{N}$ et la longitude $5^{\circ} 03^{\prime} \mathrm{O}$ (Figure 1). C'est une zone de transition forêt/savane. La végétation forestière semi-décidue est constituée de Ceiba pentadra et de Chromolena odorata alors que la savane est dominée essentiellement par les rôniers (Borassus aethiopium) et de plusieurs espèces de poacées (Comoé, 2001). Les sols de la forêt présentent une texture argilo sableuse alors que ceux de la savane ont une texture sablo argileuse. La minéralisation de la matière organique est plus élevée en forêt qu'en savane (Gianettoni, 2001). Le climat est de type équatorial de transition avec une grande saison de pluie de mars à juillet et une petite saison d'août à octobre. Ces deux saisons de pluie sont intercalées par deux saisons sèches. Les précipitations moyennes annuelles sont de $1250 \mathrm{~mm}$ de pluie reparties sur 5 à 6 mois.

\section{Matériel}

Le matériel végétal est constitué des tubercules de deux variétés locales de $D$. alata (Bètè-bètè et Florido) et de deux (2) variétés locales de $D$. cayenensis-rotundata (Krenglè et Lokpa), cultivées en Côte d'Ivoire. Les échantillons de feuilles, de tiges et de tubercules des plantes présentant les symptômes de viroses ont été utilisés. Un kit TAS-ELISA, contenant les immunoglobulines (IgG), les anticorps monoclonaux (Mab) et leurs conjugués (RaM-ap) dirigés contre le YMV (AS-0176-0435/10) et contre le CMV (AS-0475-0491/1), et un kit DAS-ELISA (IgG et son conjugué IgG-ap pour le CMV) ont été utilisés pour la détection des particules virales. Ces anticorps et le contrôle positif de l'antigène ont été fournis par la société allemande DSMZ (Plant virus collection. Mascheroder Weg 1b 38124 Braunschweig, Germany).

\section{Méthodes \\ Mise en place des parcelles paysannes.}

Les parcelles ont été défrichées, brûlées et dessouchées. L'écartement entre les buttes est de $0,5 \mathrm{~m}$ sur la ligne et de $1 \mathrm{~m}$ entre les lignes. Les variétés ont été réparties de manière aléatoire dans les parcelles. Le système de culture a été librement choisi par les paysans. L'igname est produite soit en monoculture, soit en polyculture. La monoculture de l'igname est multivariétale et polyspécifique. Dans le système polycultural, les ignames partagent la surface de champ avec des légumes (gombo, tomate, aubergine...) et des cultures vivrières telles que le manioc, la banane plantain et le maïs.

Symptomatologie et échantillonnage des plantes infectées

A 3, 5 et 7 mois après la plantation, les différents symptômes du YMV et du CMV ont été observés et décrits. Dans chacune des zones de Toumodi et de Bouaké, trois (3) localités ont été choisies de manière aléatoire. Un village par localité et six (6) parcelles par village ont été retenus pour cette étude, soit au total dix huit (18) parcelles visitées. Chaque parcelle a été subdivisée en quatre parties rectangulaires égales. Dans chacune de ces parties, quatre (4) échantillons dont deux (2) provenant des feuilles, un fragment de tige et un provenant de tubercule de deux (2) plantes présentant des symptômes caractéristiques du YMV et du CMV ont été collectées pour des analyses au laboratoire.

\section{Identification des virus}

L'identification des virus s'est faite selon la méthode de Clark et Adams (1977). Cent milligrammes de feuilles ont été broyés dans un mortier stérile puis récupérés dans 5 $\mathrm{ml}$ de tampon PBS-Tween suivi d'une centrifugation à $4000 \mathrm{rpm}$ pendant $8 \mathrm{~min}$. Les anticorps $\mathrm{IgG}, \mathrm{Mab}$ et RaM-ap dirigés contre le YMV (AS-0176-0435/10) et contre le CMV (AS-0475-0491/1) ont été utilisés pour le test TAS-ELISA. Pour les tests de confirmation du CMV, en plus du TAS-ELISA la variante Double sandwich Antibody (DAS) du même test immunoenzymatique a été réalisée. L'anticorps IgG anti CMV et son conjugué (Anti CMV-ap conjugué à la phosphatase alcaline) ont également servi à la réalisation du test DAS-ELISA. L'absorbance est lue à $405 \mathrm{~nm}$ à l'aide du lecteur de plaque ELISA, Multiskan EX Labsystems version 1.1 (Finland), après 1 heure d'incubation de la préparation à l'obscurité à $25^{\circ} \mathrm{C}$. Le seuil de positivité est le double de la moyenne des échantillons non infectés. 


\section{Distribution du YMV et CMV}

Les foyers d'infection du YMV et du CMV ont été déterminés sur toutes la surface de la parcelle.

Sur la base de la distribution de ces foyers sur la parcelle, plusieurs types de foyers ont été observés.

\section{Incidence du YMV et du CMV}

A 3,5 et 7 mois après la plantation, le pourcentage de plantes infectées par rapport au nombre total de plantes dans la parcelle a été calculé par comptage des plantes présentant des symptômes du YMV et du CMV dans chacune des localités et zone de production. La présence du YMV et du CMV a été confirmée par les tests ELISA.

Analyse statistique des données

Les données ont été analysées en utilisant un modèle linéaire généralisé (GLM) du logiciel SAS (1999). Une analyse de variance (ANOVA) a été faite pour vérifier la corrélation spatiale, spécifique et variétale de l'incidence de ces virus.

\section{RESULTATS}

\section{Symptomatologie des plantes étudiées}

Une large gamme de symptômes du YMV et du CMV a été observée aussi bien sur les variétés $D$. cayenensis-rotundata que sur D. alata. Ces symptômes sont les mosaïques, les chloroses, les feuilles en lacet de chaussures (shoestring) et les feuilles gaufrées. La mosaïque se manifeste par des mouchetures, des cloques, des ponctuations régulières ou encore des liserés des nervures. Les chloroses ont été observées sur une ou des parties de la feuille et parfois sur la tige. Les feuilles transformées en lacets de chaussures présentent une réduction très prononcée du limbe et les feuilles se vrillent. Enfin, les feuilles gaufrées, observées uniquement sur $D$. cayenensis rotundata, se présentent comme des feuilles dont les bords ressemblent à un coquillage.

\section{Identification des virus responsables des symptômes observés}

Les tests ELISA effectués sur les échantillons de feuilles, de tiges et de tubercules ont confirmé que la mosaïque, le shoestring et le gaufrage des feuilles sont causés par le YMV. Toutes les variétés locales se sont révélées infectées par le YMV avec un taux d'incidence très élevé (Tableau 1). La chlorose quant à elle, est provoquée par le CMV. L'incidence du CMV est faible avec le test TAS-ELISA par contre elle est très élevé avec le test DAS-ELISA. Par ailleurs, aucun symptôme de CMV n'a été observé sur la variété Krenglè (D. cayenensis). Des infections mixtes du YMV et CMV ont été enregistrées sur $04,10 \%$ des échantillons (Tableau 1).

\section{Evolution des symptômes du YMV et du CMV à Bouaké et à Toumodi}

Aussi bien à Bouaké qu'à Toumodi, le nombre de plantes présentant la mosaïque et le gaufrage, 2 symptômes de la manifestation du YMV, augmente avec l'âge de la plantation. Cette évolution du nombre de plantes infectées a été observée sur toutes les variétés locales pour la mosaïque et uniquement sur les variétés de $D$. cayenensis-rotundata pour le gaufrage. Aucune variété de $D$. alata infectées par le YMV n'a présenté des feuilles gaufrées. Contrairement aux 2 premiers symptômes du YMV, le nombre de plantes présentant le shoestring diminue avec l'âge de la plantation (Tableau 2 et 3 ). Les analyses statistiques ont montré qu'il existe des différences significatives entre le nombre de plantes présentant ces 3 symptômes du YMV à différents stades de la plantation (Tableau 2 et 3).

Le nombre de plantes dont les feuilles et les tiges présentent une chlorose très prononcée évolue sensiblement avec l'âge de la plantation. En outre, aucune plante de la variété Krenglè n'a manifesté de chlorose, expression du CMV, (Tableau 2 et 3). Les analyses statistiques n'ont révélé aucune différence significative entre le nombre de plantes présentant la chlorose à différents stades de la plantation (Tableau 2 et 3 ).

\section{Distribution du YMV et du CMV}

Le YMV et le CMV sont régulièrement distribués dans les 2 zones de production. Les symptômes de viroses ne sont pas uniformément repartis sur toute l'étendue de la parcelle. Le YMV présente 75,12 $\pm 10,58 \%$ de foyers marginaux en bordure des parcelles; $14,70 \pm 05,03 \%$ de foyers généralisés et 
Tableau 1: Incidence du Yam Mosaic Virus (YMV) et du Cucumber Mosaic virus (CMV) selon les tests ELISA sur les variétés de Dioscorea spp.

\begin{tabular}{|c|c|c|c|c|c|}
\hline \multirow{2}{*}{ Espèces } & \multirow{2}{*}{ Variétés } & \multirow{2}{*}{ Symptômes } & \multirow{2}{*}{$\begin{array}{c}\text { Incidence } \\
(\%)\end{array}$} & \multicolumn{2}{|c|}{ Tests ELISA } \\
\hline & & & & YMV & CMV \\
\hline \multirow{9}{*}{ D. alata } & \multirow{4}{*}{ Florido } & Mosaïque & 47,59 & ++ & - \\
\hline & & Mosaïque + Shoestring & 30,41 & + & - \\
\hline & & Shoestring+ Chlorose & 04,43 & ++ & + \\
\hline & & Chlorose & 17,67 & - & + \\
\hline & \multirow{5}{*}{ Bètè-bètè } & Mosaïque & 76,16 & +++ & - \\
\hline & & Shoestring & 07,80 & + & - \\
\hline & & Mosaïque + Shoestring & 12,00 & ++ & - \\
\hline & & Chlorose & 09,79 & - & + \\
\hline & & Mosaïque + Chlorose & 03,58 & + & + \\
\hline \multirow{3}{*}{ D. cayenensis- } & \multirow{3}{*}{ Krenglè } & Mosaïque & 52,46 & + & - \\
\hline & & Shoestring & 09,31 & + & - \\
\hline & & Gaufrage & 37,24 & + & - \\
\hline \multirow{5}{*}{ rotundata } & \multirow{5}{*}{ Lokpa } & Mosaïque & 48,28 & + & - \\
\hline & & Shoestring & 12,53 & + & - \\
\hline & & Gaufrage & 27,76 & + & - \\
\hline & & Chlorose & 07,62 & - & + \\
\hline & & Mosaïque + Chlorose & 04,65 & + & + \\
\hline
\end{tabular}

Tableau 2: Evolution de l'incidence des maladies causées par le Yam Mosaic Virus (YMV) et le Cucumber Mosaic virus (CMV) sur des variétés locales de D. alata et D. cayenensis-rotundata de Bouaké.

\begin{tabular}{|c|c|c|c|c|c|c|c|c|}
\hline \multirow[b]{4}{*}{ Virus } & \multirow[b]{4}{*}{ Sy } & \multirow[b]{4}{*}{ MAP } & \multicolumn{4}{|c|}{ Espèces } & \multirow{4}{*}{$\mathbf{F}$} & \multirow{4}{*}{$\mathbf{P}$} \\
\hline & & & \multirow{2}{*}{\multicolumn{2}{|c|}{$\begin{array}{l}\text { D. alata } \\
\text { Variétés }\end{array}$}} & \multirow{2}{*}{\multicolumn{2}{|c|}{$\begin{array}{c}\text { D.cayenensis-rotundata } \\
\text { Variétés }\end{array}$}} & & \\
\hline & & & & & & & & \\
\hline & & & Bètè-bètè & Florido & Lokpa & Krenglè & & \\
\hline \multirow{9}{*}{ YMV } & \multirow{3}{*}{ M } & 3 & $50,51 \pm 05,30 \mathbf{c}$ & $37,25 \pm 02,36 \mathbf{c}$ & $38,87 \pm 02,05 \mathbf{c}$ & $31,17 \pm 01,68 \mathbf{c}$ & \multirow{3}{*}{63,43} & \multirow{3}{*}{$<0.0001$} \\
\hline & & 5 & $79,12 \pm 06,20 \mathbf{b}$ & $69,76 \pm 04,94 \mathbf{b}$ & $57,22 \pm 02,58 \mathbf{b}$ & $50,15 \pm 02,65 \mathbf{b}$ & & \\
\hline & & 7 & $92,33 \pm 05,20 \mathbf{a}$ & $88,13 \pm 05,40 \mathbf{a}$ & $85,68 \pm 03,57 \mathbf{a}$ & $80,9 \pm 03,04 \mathbf{a}$ & & \\
\hline & \multirow{3}{*}{ Sh } & 3 & $20,56 \pm 04,40 \mathbf{a}$ & $12,68 \pm 02,85 \mathbf{a}$ & $19,78 \pm 03,2 \mathbf{a}$ & $26,36 \pm 04,18 \mathbf{a}$ & \multirow{3}{*}{14,59} & \multirow{3}{*}{$<0.0001$} \\
\hline & & 5 & $11,07 \pm 01,90 \mathbf{b}$ & $08,18 \pm 01,98 \mathbf{b}$ & $11,25 \pm 02,7 \mathbf{b}$ & $17,54 \pm 02,47 \mathbf{b}$ & & \\
\hline & & 7 & $06,11 \pm 01,70 \mathbf{c}$ & $04,55 \pm 01,38 \mathbf{c}$ & $08,03 \pm 02,19 \mathbf{c}$ & $07,34 \pm 02,23 \mathbf{c}$ & & \\
\hline & \multirow{3}{*}{$\mathrm{G}$} & 3 & 00 & 00 & $12,43 \pm 03,50 \mathbf{c}$ & $10,30 \pm 01,87 \mathbf{c}$ & \multirow{3}{*}{11,94} & \multirow{3}{*}{$<0.0001$} \\
\hline & & 5 & 00 & 00 & $25,01 \pm 01,92 \mathbf{b}$ & $19,41 \pm 02,44 \mathbf{b}$ & & \\
\hline & & 7 & 00 & 00 & $36,00 \pm 02,6 \mathbf{c}$ & $30,14 \pm 04,38 \mathbf{c}$ & & \\
\hline \multirow{3}{*}{ CMV } & \multirow{3}{*}{$\mathrm{C}$} & 3 & $08,05 \pm 00,40 \mathbf{c}$ & $06,04 \pm 00,01 \mathbf{c}$ & $05,60 \pm 00,07 \mathbf{c}$ & 00 & \multirow{3}{*}{09,04} & \multirow{3}{*}{0.1813} \\
\hline & & 5 & $10,50 \pm 00,72 \mathbf{b}$ & $08,50 \pm 00,15 \mathbf{b}$ & $06,78 \pm 00,36 \mathbf{b}$ & 00 & & \\
\hline & & 7 & $14,08 \pm 00,64 \mathbf{a}$ & $10,00 \pm 00,30 \mathbf{a}$ & $09,05 \pm 00,04 \mathbf{a}$ & 00 & & \\
\hline
\end{tabular}

y: Symptômes, M: Mosaïque, Sh: Shoestring, Gaufrage, G: Chlorose. Suivant la colonne, les taux d'incidence moyens portant la même lettre ne présentent pas de différence statistiquement significative (LDS ; P $=0,05$ ) selon le test ANOVA II MAP : Mois Après Plantation. 
Tableau 3: Evolution de l'incidence des maladies causées par le Yam Mosaic Virus (YMV) et le Cucumber Mosaic virus (CMV) sur des variétés locales de D. alata et D. cayenensis-rotundata de Toumodi.

\begin{tabular}{|c|c|c|c|c|c|c|c|c|}
\hline \multirow[b]{3}{*}{ Virus } & \multirow[b]{3}{*}{ Sy } & \multirow[b]{3}{*}{ MAP } & \multicolumn{4}{|c|}{ Espèces } & \multirow{3}{*}{$\mathbf{F}$} & \multirow{3}{*}{$\mathbf{P}$} \\
\hline & & & \multicolumn{2}{|c|}{\begin{tabular}{|l|} 
D. alata \\
Variétés \\
\end{tabular}} & \multicolumn{2}{|c|}{$\begin{array}{c}\text { D.cayenensis-rotundata } \\
\text { Variétés } \\
\end{array}$} & & \\
\hline & & & Bètè-bètè & Florido & Lokpa & Krenglè & & \\
\hline \multirow{9}{*}{ YMV } & \multirow{3}{*}{$\mathrm{M}$} & 3 & $56,05 \pm 05,22 \mathrm{c}$ & $45,22 \pm 3,59 \mathbf{c}$ & $40,95 \pm 02,30 \mathbf{c}$ & $38,70 \pm 01,82 \mathbf{c}$ & \multirow{3}{*}{72,37} & \multirow{3}{*}{$<.0001$} \\
\hline & & 5 & $87,62 \pm 04,01 \mathbf{b}$ & $75,93 \pm 05,54 \mathbf{b}$ & $70,36 \pm 04,80 \mathbf{b}$ & $62,18 \pm 05,33 \mathbf{b}$ & & \\
\hline & & 7 & $98,00 \pm 07,01 \mathbf{a}$ & $92,05 \pm 6,19 \mathbf{a}$ & $86,25 \pm 04,74 \mathbf{a}$ & $84,21 \pm 04,74 \mathbf{a}$ & & \\
\hline & \multirow{3}{*}{ Sh } & 3 & $22,27 \pm 03,43 \mathbf{a}$ & $18,81 \pm 02,26 \mathbf{a}$ & $35,99 \pm 03,97 \mathbf{a}$ & $25,33 \pm 04,24 \mathbf{a}$ & \multirow{3}{*}{11,60} & \multirow{3}{*}{$<.0001$} \\
\hline & & 5 & $19,82 \pm 03,09 \mathbf{b}$ & $11,13 \pm 01,92 \mathbf{b}$ & $25,77 \pm 03,89 \mathbf{b}$ & $20,90 \pm 02,94 \mathbf{b}$ & & \\
\hline & & 7 & $09,50 \pm 02,71 \mathbf{c}$ & $06,34 \pm 02,43 \mathbf{c}$ & $17,03 \pm 02,79 \mathbf{c}$ & $10,30 \pm 02,01 \mathbf{c}$ & & \\
\hline & \multirow{3}{*}{$\mathrm{G}$} & 3 & 00 & 00 & $18,33 \pm 04,35 \mathbf{c}$ & $12,22 \pm 03,51 \mathrm{c}$ & \multirow{3}{*}{09,43} & \multirow{3}{*}{$<.0001$} \\
\hline & & 5 & 00 & 00 & $26,70 \pm 05,44 \mathbf{b}$ & $20,65 \pm 02,59 \mathbf{b}$ & & \\
\hline & & 7 & 00 & 00 & $40,57 \pm 03,95 \mathbf{a}$ & $37,12 \pm 03,15 \mathbf{a}$ & & \\
\hline \multirow{3}{*}{ CMV } & \multirow{3}{*}{$\mathrm{C}$} & 3 & $11,50 \pm 03,30 \mathbf{c}$ & $08,16 \pm 00,02 \mathbf{c}$ & $07,44 \pm 00,07 \mathbf{c}$ & 00 & \multirow{3}{*}{15,27} & \multirow{3}{*}{0.0026} \\
\hline & & 5 & $15,02 \pm 00,08 \mathbf{b}$ & $13,40 \pm 00,09 \mathbf{b}$ & $10,30 \pm 00,11 \mathbf{b}$ & 00 & & \\
\hline & & 7 & $22,50 \pm 00,44 \mathbf{a}$ & $15,97 \pm 00,05 \mathbf{a}$ & $13,01 \pm 00,05 \mathbf{a}$ & 00 & & \\
\hline
\end{tabular}

NB: Sy: Symptômes, M: Mosaïque, Sh: Shoestring, Gaufrage, G: Chlorose. Suivant la colonne, les taux d'incidence moyens portant la même lettre ne présentent pas de différence significative (LDS; $\mathrm{P}=0,05$ ) selon le test ANOVA II MAP : Mois Après Plantation.

$11,86 \pm 03,96 \%$ de foyers centraux dans la zone de Toumodi. A Bouaké 62,00 $\pm 07,72 \%$ des foyers sont marginaux, $15,40 \pm 06,21 \%$ des foyers centraux et $23,31 \pm 05,44 \%$ des infections généralisées. Le YMV est présent sur toutes les variétés. Le CMV est faiblement distribué dans les localités des 2 zones de production. Les foyers d'infection sont $55,23 \pm 08,57 \%$ de types marginaux et $45,77 \pm 09,41 \%$ isolés dans les parcelles de Toumodi. Le CMV est absent sur la variété Krenglè. A Bouaké comme à Toumodi, les infections du YMV et du CMV sont dominées par des foyers marginaux suivis des foyers généralisés (Tableau 4).

\section{Relation entre incidence du YMV et du CMV et variété}

Toutes les variétés locales de Dioscorea spp. ont été infectées par le YMV. L'incidence du YMV est très élevée aussi bien sur les variétés Bètè-bètè et Florido de l'espèce $D$. alata (respectivement $97,06 \pm 11,23 \%$ et $95,04 \pm 05,06 \%$ ) que sur les variétés Lokpa et Krenglè de $D$. cayenensisrotundata (respectivement 93,36 $\pm 03,66 \%$ et $91,22 \pm 04,82 \%)$. Les analyses statistiques ont révélé des différences entre les variétés (Tableau 5).

Des faibles incidences du CMV ont été enregistrées sur les variétés Bètè-bètè, Florido et Lokpa. Aucune plante de la variété Krenglè (D. cayenensis) n'a été infectée par le CMV. Sur l'ensemble des deux (2) zones de production, l'espèce $D$. alata s'est avérée plus infectée par le YMV que l'espèce $D$. cayenensis-rotundata (Tableau 5). Des différences statistiquement significatives existent entre les variétés infectées par le CMV (Tableau 5).

\section{Incidence du YMV et du CMV et zone de production}

Le YMV a une forte incidence dans la zone de Bouaké et de Toumodi, avec respectivement $88 \%$ et $95 \%$ de plantes infectées (Tableau 6). Quant aux CMV, une faible incidence de ce virus a été observée dans la zone de production de Bouaké et de Toumodi avec respectivement $10,85 \%$ et $16,28 \%$ (Tableau 6). L'analyse statistique a relevé des différences hautement significatives entre ces deux (2) zones de production $(\mathrm{p} \leq 0,0001)$. 


\section{DISCUSSION}

Les tests sérologiques effectués sur les échantillons de feuilles, de tiges, et de tubercules ont confirmé que la mosaïque, le gaufrage et le shoestring sont causées par les YMV. Les chloroses observées sont, quant à elles, les symptômes du CMV. Les infections de ces deux (2) virus sont de types marginaux ou généralisés. La transmission de ces virus se fait par les aphides selon le mode non persistant et par les tubercules infectés. Ces insectes infectent les plantes à partir des réservoirs d'inoculum situés en bordure des parcelles. C'est ce qui explique les taux élevés d'infections marginales. Les infections généralisées proviendraient de certains tubercules infectés distribués sur toute l'étendue de la parcelle. Le YMV a été observé aussi bien sur les variétés de $D$. alata que sur les variétés de $D$. cayenensisrotundata dans chacune des localités des

Tableau 4: Distribution du Yam Mosaic Virus (YMV) et du Cucumber Mosaic virus (CMV) dans les parcelles de Toumodi et de Bouaké.

\begin{tabular}{|c|c|c|c|c|c|c|}
\hline \multirow{2}{*}{$\begin{array}{l}\text { V } \\
\text { Fy } \\
Z\end{array}$} & \multicolumn{3}{|c|}{ YMV } & \multicolumn{3}{|c|}{ CMV } \\
\hline & $\begin{array}{l}\text { Foyer } \\
\text { marginal }\end{array}$ & $\begin{array}{l}\text { Foyer } \\
\text { central }\end{array}$ & $\begin{array}{l}\text { Foyer } \\
\text { généralisé }\end{array}$ & $\begin{array}{l}\text { Foyer } \\
\text { marginal }\end{array}$ & $\begin{array}{l}\text { Foyer } \\
\text { central }\end{array}$ & $\begin{array}{l}\text { Foyer } \\
\text { généralisé }\end{array}$ \\
\hline $\mathrm{T}$ & $75,12 \pm 10,58 \mathbf{a}$ & $11,86 \pm 03,96 \mathbf{d}$ & $14,7 \pm 05,03 \mathbf{d}$ & $55,23 \pm 08,6 \mathbf{a b}$ & $10,28 \pm 02,45 \mathbf{d}$ & $35,77 \pm 09,4 \mathbf{b}$ \\
\hline B & $62,0 \pm 07,7 \mathbf{a b}$ & $15,40 \pm 06,21 \mathbf{d}$ & $23,31 \pm 05,44 \mathbf{c}$ & $88,13 \pm 09,6 \mathbf{a}$ & $03,53 \pm 01,64 \mathbf{e}$ & $08,44 \pm 0,9 \mathbf{d}$ \\
\hline $\mathrm{F}$ & & 77.13 & & & 190.06 & \\
\hline $\mathrm{P}$ & & $<.0001$ & & & $<.0001$ & \\
\hline
\end{tabular}

Tableau 5: Incidence des viroses causées par Yam Mosaic Virus (YMV) et Cucumber Mosaic virus (CMV) sur les variétés locales de D. alata et $D$. cayenensis-rotundata.

\begin{tabular}{|c|c|c|c|c|c|c|c|}
\hline \multirow{2}{*}{\multicolumn{2}{|c|}{$\begin{array}{l}\text { Virus } \\
\text { observés }\end{array}$}} & \multicolumn{2}{|c|}{$\frac{\text { D. alata }}{\text { Variété }}$} & \multicolumn{2}{|c|}{$\begin{array}{c}\text { D. cayenensis-rotundata } \\
\text { Variété }\end{array}$} & \multirow{2}{*}{$\mathbf{F}$} & \multirow{2}{*}{$\mathbf{P}$} \\
\hline & & Bètè-bètè & Florido & Lopka & Krenglè & & \\
\hline YMV & $\begin{array}{l}\text { Min. } \\
\text { Moy. } \\
\text { Max. }\end{array}$ & $\begin{array}{c}79,20 \\
97,06 \pm 11,23 \mathbf{a} \\
99,14\end{array}$ & $\begin{array}{c}68,52 \\
95,04 \pm 05,06 \mathbf{b} \\
97,41\end{array}$ & $\begin{array}{c}60,4 \\
93,38 \pm 03,66 \mathbf{c} \\
95,74\end{array}$ & $\begin{array}{c}59,5 \\
91,22 \pm 04,82 \mathbf{d} \\
93,83\end{array}$ & 346,10 & $<.0001$ \\
\hline CMV & $\begin{array}{l}\text { Min. } \\
\text { Moy. } \\
\text { Max. }\end{array}$ & $\begin{array}{c}10,00 \\
18,07 \pm 01,43 \mathbf{a} \\
22,58\end{array}$ & $\begin{array}{c}07,30 \\
12,16 \pm 02,72 \mathbf{b} \\
15,35\end{array}$ & $\begin{array}{c}06,47 \\
09,23 \pm 01,08 \mathbf{c} \\
13,84\end{array}$ & 00 & 09,32 & $<.0001$ \\
\hline
\end{tabular}

Min : minimal; Moy : moyenne; Max: maximal. Sur la ligne les taux d'incidence portant les mêmes lettres ne présentent pas de différences statistiquement significatives (LDS; P = 0,05) selon le test ANOVA II.

Tableau 6: Comparaison de l'incidence du Yam Mosaic Virus (YMV) et Cucumber Mosaic virus (CMV) sur les variétés locales dans les zones de Bouaké et de Toumodi.

\begin{tabular}{|c|c|c|c|c|c|c|c|c|}
\hline Virus & & & & & & CMV & & \\
\hline $\mathrm{VZ}$ & Bètè-bètè & Florido & Lopka & Krenglè & Bètè-bètè & Florido & Lopka & $\mathrm{K}$ \\
\hline B & $93,21 \pm 8,04 \mathbf{b}$ & $90,51 \pm 5,13 \mathbf{b}$ & $87,74 \pm 7,21 \mathbf{b}$ & $83,21 \pm 3,74 \mathbf{b}$ & $15,20 \pm 2,56 \mathbf{b}$ & $09,82 \pm 2,23 \mathbf{b}$ & $07,46 \pm 1,27 \mathbf{b}$ & 00 \\
\hline $\mathrm{T}$ & $98,35 \pm 6,36 \mathbf{a}$ & $96,63 \pm 3,41 \mathbf{a}$ & $94,52 \pm 2,96 \mathbf{a}$ & $92,39 \pm 5,77 \mathbf{a}$ & $19,37 \pm 3,11 \mathbf{a}$ & $15,15 \pm 1,28 \mathbf{a}$ & $13,02 \pm 2,39 \mathbf{a}$ & 00 \\
\hline $\mathrm{F}$ & 139,08 & 113,91 & 125,58 & 104,6 & 57,23 & 38,10 & 26,90 & - \\
\hline $\mathrm{P}$ & $<.0001$ & $<.0001$ & $<.0001$ & $<.0001$ & $<.0001$ & $<.0001$ & $<.0001$ & - \\
\hline
\end{tabular}


zones de Bouaké et de Toumodi. L'incidence du YMV varie en fonction de l'espèce (Eni et al., 2008 a) et de l'état phénologique de la plante. Des observations similaires ont été faites par Thottappily (1992) et Lebas (2002). Le YMV, autrefois, observé uniquement dans la région de Bouaké (Thouvenel et Fauquet, 1979; Thouvenel et Dumont, 1988) s'est répandu également dans toute la région deToumodi. Ce virus est présent dès les premiers stades du développement de la culture et son incidence augmente avec le stade phénologique de la plante. L'incidence du YMV diminue de la zone de transition forêt/savane (Toumodi) à la zone de savane (Bouaké). Ces résultats sont similaires à ceux Thouvenel et Fauquet, (1979) qui ont enregistré une augmentation de l'incidence du YMV et du CMV en partant de la frontière du Burkina (savane) au sud de la Côte d'Ivoire (forêt). Les symptômes de shoestring régressent avec l'âge de la plantation. Ces mêmes résultats ont été obtenus sur les variétés améliorées TDr 87 / 02461 et sur TDa 95 / 00010 par Kouamé et al. (2003). Certaines plantes pourraient développer un phénomène de résistance au contact du YMV. Il est également possible qu'un autre variant du YMV dont le pouvoir infectieux diminue avec le temps soit présent. Le gaufrage des feuilles observé uniquement sur $D$. cayenensis-rotundata, découvert pour la première fois à N'douci, dans le sud forestier de la Côte d'Ivoire (Thouvenel et Fauquet, 1979) est maintenant observé dans les zones de production de Bouaké et de Toumodi. Cependant, les symptômes de gaufrage n'ont pas été observés sur les variétés de $D$. alata probablement grâce à leur cuticule épaisse. Le YMV constitue une menace réelle puisqu'il a une incidence élevée aussi bien à Toumodi qu'à Bouaké, sur toutes les espèces et variétés et durant tout le stade phénologique de la culture. Ces résultats sont conformes à ceux de Thouvenel et Fauquet (1990), GoudouUrbino et al. (1996) et Séka et al. (2009) qui ont affirmé que le YMV est un facteur très limitant pour la culture de l'igname. Les variétés de $D$. alata, les plus cultivées, présentent les plus fort taux de mosaïque. C'est à juste titre que Bousalem et al. (1997) ont affirmé que les $D$. alata sont généralement tolérantes à la mosaïque de l'igname. Sur $D$. cayenensis-rotundata, une forte incidence de shoestring et de gaufrage de feuilles a été enregistrée. La forme étroite des feuilles de $D$. cayenensis-rotundata est une prédisposition à l'apparition du shoestring et du gaufrage des feuilles. Le nombre de plante infectées par le YMV et dont les symptômes se manifestent par la mosaïque et le gaufrage des feuilles augmentent avec l'âge de la parcelle quelle que soit la variété de Dioscorea spp.cultivée. Cela s'explique par le fait que dans la plupart des cas, le YMV et le CMV sont transmis par les insectes piqueur-suceurs selon le mode non persistant. Ainsi, plusieurs plantes peuvent être infectées par le même vecteur en une seconde. Dans la zone de Toumodi (zone de transition forêt / savane), les symptômes de YMV autres que le shoestring sont très présents dans la localité située dans la partie savanicole (Toumodi) que dans la partie forestière (Kocoumbo). Ces résultats sont similaires à ceux de Gumedzoe et al. (2003) qui ont révélé que le taux de prévalence et l'incidence du virus de la mosaïque de l'igname diffèrent d'une région à une autre au Togo. Le CMV est faiblement distribué et réparti dans les parcelles, localités et zones d'étude. Sur 1152 échantillons présentant les symptômes caractéristiques de ces deux viroses, seulement $10 \%$ présentaient une infection au CMV. En outre, aucune infection de CMV n'a été observée naturellement sur la variété Krenglè. Ces faibles incidences du CMV ont été également observées par Eni et al. (2008b). Cet auteur a observé 2,75\% d'échantillons infectés par le CMV au Togo, $2,07 \%$ au Ghana et $0,71 \%$ au Bénin. Cela justifie les conclusions des travaux de Tokpa et al. (1998) qui préconisent une sélection au sein de la variété Krenglè pour réduire les infections virales. Il serait intéressant de réaliser des inoculations de la variété Krenglé avec le CMV pour déterminer la réaction de ces variétés aux CMV. Des études complémentaires doivent être également faites sur d'autres variétés de D. cayenensisrotundata et dans d'autres zones de production pour apprécier le comportement naturel de ces variétés vis-à-vis du CMV. Bien que des études antérieures aient montré la susceptibilité de certaines variétés locales 
(Thouvenel et Fauquet, 1990), ce manuscrit relate pour la première fois la sensibilité de la variété locale Lokpa au YMV et au CMV. La présence du YMV et du CMV dans certains échantillons n'a pu être confirmé par les tests ELISA bien qu'ils présentaient des symptômes de ces deux (2) viroses. Cela s'explique soit par le fait que le titre viral de ces échantillons était trop faible pour être détecté par les TAS ELISA, soit par la présence d'un autre virus présentant des symptômes similaires au YMV et au CMV. Les résultats que nous rapportons dans ce papier constituent les premiers cas signalés en Côte d'Ivoire, d'infections mixtes impliquant YMV et CMV sur les variétés Bètè-bètè, Florido et Lokpa. Ces infections mixtes entraînent souvent des symptômes très sévères (Diallo et al., 2004) avec la détérioration des tubercules d'ignames.

\section{Conclusion}

Les présents travaux confirment la présence du YMV et du CMV dans les zones de Toumodi et de Bouaké. Toutes les variétés locales sont infectées par le YMV avec une très forte incidence dans chacune de ces deux (2) grandes zones de production d'ignames en Côte d'Ivoire et se manifeste par la mosaïque, le shoestring, et le gaufrage des feuilles. Le shoestring, causé par le YMV, diminue chez la plante au fur et à mesure que le stade phénologique de la plante évolue. Le CMV moins présent sur Dioscorea spp. dans ces deux (2) grandes zones de production se distingue par des chloroses très sévères. Il a été observé sur toutes les variétés locales autres que Krenglè (Dioscorea cayenensis). Le comportement d'autres variétés de Dioscorea cayenensis vis-à-vis du CMV devait être étudié dans différentes zones de production d'ignames en Côte d'Ivoire. Des infections mixtes impliquant YMV et CMV ont été observées dans les deux (2) zones de production. Il s'agit du premier rapport d'infections mixtes impliquant YMV et CMV sur les variétés Bètè-bètè, Florido et Lokpa en Côte d'Ivoire. Ces infections mixtes à interactions synergétiques entraînent souvent des symptômes très sévères avec la détérioration des tubercules d'ignames. Les techniques culturales associées à l'utilisation de semences saines pourraient contribuer à réduire ces infections virales et constituer l'une des meilleures manières de lutter contre le YMV et le CMV.

\section{REMERCIEMENTS}

Ces travaux ont pu être réalisés grâce soutien financier de la Fondation IFSCORAF.

\section{REFERENCES BIBLIOGRAPHIQUES}

Asiedu R, Ng SYC, Bai KV, Ekanayake IJ, Wanyera NMW. 1998. Genetic Improvement. In Food Yams, Advances in Research, Orkwor GC, Asiedu R, Ekanayake IJ (eds). IITA and NCRI: Nigeria; 63-104.

Bousalem M, Pinel A, Dubern J, Frutos R, Fargette D. 1997. Evaluation de la variabilité moléculaire du virus de la mosaïque de l'igname. Comptes rendus des $6^{\mathrm{e}}$ rencontres de virologie végétale. Ecole thématique, délégation régionale Alsace du Cnrs, Ecole thématique, délégation régionale Alsace du Cnrs, 9-13 mars, 1997, Strasbourg, France, 72p.

Bousalem M, Douzery EJP, Fargette D. 2000. High genetic diversity, distant phylogenetic relationships and intraspecies recombination events among natural populations of Yam mosaic virus: a contribution to understanding Potyvirus evolution. Journal of General Virology, 81: 243-255.

Clark MF, Adams AN. 1977. Characteristics of the microplate method of enzymelinked immunosorbent assay for the detection of plant viruses. Journal of General Virology, 34: 475-483.

Comoé KB. 2001. Evolution de la matière sèche chez deux cultivars améliorés d'igname (Dioscorea spp.) dans 2 zones agroécologiques sous l'effet de la fumure minérale. Mémoire d'ingénieur agronome, Ecole Supérieure d'Agronomie, Yamoussoukro, Côte d'Ivoire, 21-32.

Diallo HA, Gergerich RC, Anderson EJ. 2004. Partial molecular characterization of cowpea stunt isolates of CMV and BlCMV from Arkansas and Georgia. Agronomie Africaine, 16: 59-69. 
Eni AO, Hughes Jd'A, Rey MEC. 2008a. Survey of the incidence and distribution of five viruses infecting yams in the major yam-producing zones in Benin. Annals of Applied Biology, 153: 223-232.

Eni AO, Lavar K, Asiedu R, Alabi OJ, Naidu RA, Hughes Jd'A, Rey MEC. 2008b. First report of cucumber mosaic virus in yams (Dioscorea spp.) in Ghana, Togo and Republic of Benin in West Africa. Plant Disease, 92(5): 833.

FAO. 2007. Food and Agricultural Organization Statistics Database. http:// faostat.fao.org/site/567/DesktopDefault.aspx.

Jones RAC, Coutts BA, Latham LJ, McKirdy SJ. 2008. Cucumber mosaic virus infection of chickpea stands: temporal and spatial patterns of spread and yieldlimiting potential. Plant Pathology, 57(5): 842-853.

Gianettoni D. 2001. Caractérisation morphologique et physicochimique des sols du projet Nutrition minérale de l'igname, Ecole Polytechnique Fédérale de Zurich, Suisse, 13.

Goudou-Urbino C, Givord L, Quiot JB, Boeglin M, Konaté G, Dubern J. 1996. Differentiation of yam mosaic virus isolates using symptomatology, westernblot assay, and monoclonal antibodies. $J$ phytopathol, 144: 235-240.

Gumedzoe MYD, Fontem DA, Sanwgou LY, Feteke F, Thottappilly G, Adjata G, Ayisah DK. 2003. Prévalence des virus de l'igname et incidence du virus de la mosaïque de l'igname (YMV) dans des champs de Dioscorea spp. dans les préfectures de Bassar et de Sotouboua au Togo. Sciences Agronomiques et Développement, 3(1) : 49-54.

Kouamé H, Diby L, Bah P. 2003. Influence de la mosaïque de l'igname et du shoestring sur la production de la matière sèche chez deux espèces d'igname (Dioscorea spp.) cultivés dans 2 zones agro écologiques avec différentes doses de fumures minérales. Mémoire de DAA, Ecole Supérieure d'Agronomie, 41.
Lebas BSM. 2002. Diversity of virus infecting Dioscorea species in the South Pacific. $\mathrm{PhD}$ thesis, University of Greenwich Natural Resources Institute, 302p.

Odu BO, Hughes J. d'A, Asiedu R, Shoyinka A, Oladiran OA. 2004. Responses of white yam (Dioscorea rotundata) cultivars to inoculation with three viruses. Plant Pathology, 53: 141-147.

SAS SAS/STAT. 1999. Users Guide ( $5^{\text {th }}$ Edn) version 8. SAS Institute Inc.: Cary, NC, USA.

Séka K, Diallo HA, Kouassi NK, Aké S. 2009. Screening ten yam (Dioscorea spp.) varieties for resistance to Yam mosaic virus and Cucumber mosaic virus in Côte d'Ivoire. African Journal of Plant Science and Biotechnology, 3: (in press).

Tokpa G, Dumont R. 1998. Sélection au sein du groupe d'ignames Krenglè (Dioscorea cayenensis-rotundata). Proc. 6th Symposium of the ISTRC-AB, Lilongwe (Malawi), 22-28 Oct. 1995. Ibadan, Nigeria, ISTRC-AB, IITA, 426-429.

Thottappily G. 1992. Plant virus diseases of importance to African Agriculture. Phytopathology, 134: 265-288.

Thouvenel JC, Fauquet C. 1979. Yam mosaic virus, a new potyvirus infecting Dioscorea cayenensis in the Ivoiry Coast. Annals of applied Biology, 93: 279-283.

Thouvenel JC, Dumont R. 1988. An epidemiological approach to the study of yam mosaic virus in the Ivory Coast. Proceedings of the International Society for Tropical Root Crops, October 30November 5, 1988, Bangkok, Thailand, 643-648.

Thouvenel JC, Borg-Olivier, Dumont R. 1989. Epidemiology of yam mosaic virus. Importance of Aphid transmission. IVème colloque international. Epidémiologie des virus des plantes. 3-8 septembre, 1989. Montpellier, France, 49.

Thouvenel JC, Dumont R. 1990. Pertes de rendement de l'igname infecté par le virus de la mosaïque de l'igname en Côte d'ivoire. L'Agronomie Tropicale, 45(2): 125-129. 